\title{
(2) PRDDUCᄃี̆ DNLINE

\section{ANÁLISE DAS PRINCIPAIS MÉTRICAS UTILIZADAS POR PROFISSIONAIS NA AVALIAÇÃO DA MATURIDADE DE PROJETOS DE LEAN}

\section{ANALYSIS OF THE MAIN METRICS USED BY PROFESSIONALS IN THE EVALUATION OF THE LEAN PROJECTS MATURITY}

\author{
Vivien Cielusinsky*E-mail: vivicavinato@gmail.com \\ Rosley Anholon*E-mail: rosley@fem.unicamp.br \\ Izabela Simon Rampasso*,*E-mail: izarampasso@gmail.com \\ Dirceu Silva ${ }^{* * *}$ E-mal: dirceuds@gmail.com \\ Osvaldo L. G. Quelhas** E-mail: osvaldoquelhas@id.uff.br
}

*Faculdade de Engenharia Mecânica, Universidade Estadual de Campinas (Unicamp), Campinas, SP

** Laboratório de Tecnologia, Gestão de Negócios e Meio Ambiente (Latec), Universidade Federal

Fluminense (UFF), Niterói, RJ

*** Faculdade de Educação, Universidade Estadual de Campinas (Unicamp), Campinas, SP

Resumo: O Lean Manufacturing é uma estratégia de produção aplicada em empresas de diversos segmentos. Seu principal objetivo é reduzir ao máximo os desperdícios a fim de maximizar o lucro. A transição para o referido estágio, entretanto, não é simples e muitas são as barreiras enfrentadas para se alcançar os objetivos supracitados. Nesta trajetória, as empresas apresentarão diferentes estágios de maturidade e a mensuração desta maturidade torna-se importante. Tomando por base as informações mencionadas, o presente artigo tem por objetivo identificar quais são as principais métricas utilizadas por empresas brasileiras na avaliação da maturidade de sistemas lean. Métricas utilizadas em projetos lean foram listadas a partir da literatura e informações sobre a aplicação das mesmas foram levantadas junto a 43 profissionais que participaram de uma survey. Respondentes outliers foram identificados a partir do Escalonamento Multidimensional (EMD) e, na sequência, as métricas foram ordenadas via um mecanismo utilizado pelo software SPSS para combinação linear de variáveis, empregado na Análise Fatorial Exploratória. Os resultados evidenciaram que as métricas mais aplicadas estão relacionadas à produtividade dos funcionários, número de reclamações dos consumidores, tempo de setup, lead times e índice de estoques. Os resultados decorrentes deste estudo poderão ser utilizados por outros pesquisadores em suas futuras pesquisas.

Palavras-chave: Gestão da Produção. Lean Manufacturing. Métricas para avaliação da maturidade.

Abstract: Lean Manufacturing is a production strategy applied in companies of different segments. Its main goal is to minimize waste in order to maximize profit. The transition to this stage, however, is not easy and many barriers are faced to achieve the aforementioned goals. In this trajectory, companies will present different stages of maturity and the measurement of this maturity becomes important. Based on the information mentioned, this article aims to identify which are the main metrics used by Brazilian companies in assessing the maturity of lean systems. Metrics used in lean projects were listed from the literature and information about their application was collected from 43 professionals who participated in a survey. Respondents outliers were identified from the Multidimensional Scheduling (MDS) and, subsequently, the metrics were ordered via a mechanism used by SPSS software for linear combination of variables, used in Exploratory Factor Analysis. The results showed that the most applied metrics are related to employee productivity, number of consumer complaints, setup time, lead times and stock index. The results from this study may be used by other researchers in their future research.

Keywords: Production Management. Lean Manufacturing. Metrics for Maturity Assessment. 


\section{INTRODUÇÃO}

O lean manufacturing é uma filosofia que se iniciou no Japão a partir da segunda metade do século XX, e, desde então, alterou significativamente o sistema produtivo mundial. Seu surgimento e implantação estão relacionados ao ressurgimento das empresas japonesas, que se encontravam arrasadas em decorrência da segunda guerra mundial. A Toyota se caracterizou como o principal expoente desta nova era produtiva, apesar de não ter sido a única a desenvolver a referida filosofia (ANHOLON; SANO, 2016; GHOSH, 2012; JING; NIU; CHANG, 2015; MESSAGE COSTA et al., 2018).

O principal objetivo dos sistemas lean é reduzir ao máximo os desperdícios, a fim de se obter o maior lucro possível (MARODIN et al., 2018; MESSAGE COSTA et al., 2018; UFUA; PAPADOPOULOS; MIDGLEY, 2018). Dessa forma, essa filosofia começou a ganhar destaque e empresas do mundo inteiro começaram a aplicar seus princípios e ferramentas visando obter melhores resultados. Países como Brasil (JABBOUR; JUNIOR; JABBOUR, 2014; LUCATO et al., 2014), Alemanha (LEYER; MOORMANN, 2014), Inglaterra (BHASIN, 2011), Índia (GHOSH, 2012), Hungria (GELEI; LOSONCI; MATYUSZ, 2015), Tailândia (KHANCHANAPONG et al., 2014) e Sri Lanka (SILVA; PERERA; SAMARASINGHE, 2012) possuem destaques em pesquisas e estudos de caso relacionados ao tema.

Apesar dessa filosofia proporcionar ganhos significativos, a transição de sistemas convencionais de produção para o sistema lean não é imediata e não produz os resultados esperados automaticamente (BHASIN, 2012; ZHOU, 2016). Trata-se de um processo lento, que requer comprometimento de todos os envolvidos. Além disso, existem barreiras que devem ser enfrentadas para se atingir os objetivos pretendidos (BABALOLA; IBEM; EZEMA, 2018; ZHOU, 2016).

De acordo com a literatura, as maiores dificuldades existentes na implantação do lean são: custos de investimento, falta de entendimento do processo e de seus possíveis benefícios, dificuldade na implantação das ferramentas lean de forma eficaz, falta de comunicação entre os trabalhadores a respeito de mudanças no processo, treinamento e liderança para o alcance das metas (BHASIN, 2012; JADHAV; MANTHA; RANE, 2014).

Em função das dificuldades supramencionadas, é notório que diferentes empre- 
sas apresentem diferentes graus de implantação lean, ou seja, algumas se encontram em níveis mais avançados do que outras. Apesar desta filosofia ser bastante conhecida, ainda são poucas as empresas que conseguiram implantar seus princípios de forma eficiente, evidenciando-se assim, amplas possibilidades de pesquisas que tenham como foco o alcance de melhores resultados (BHASIN, 2012; ZHOU, 2016).

Mediante ao exposto, pode-se dizer que estudos associados ao grau de maturidade de empresas que atuam segundo a filosofia lean são de grande valia e pouco explorados. Com o intuito de contribuir com a temática, este artigo almeja realizar uma revisão sistemática da literatura a fim de estruturar uma lista com as principais métricas utilizadas para avaliar a maturidade de sistemas lean e, na sequência, realizar uma survey junto a profissionais do mercado para verificar quais delas são as mais aplicadas. Não se pretende estabelecer um modelo para avaliação da maturidade lean, mas sim entender como profissionais estão atuando na medição da maturidade de seus sistemas.

Além desta seção introdutória, este artigo apresenta mais quatro seções. A seção dois apresenta a fundamentação teórica dos principais temas aqui abordados, a seção três detalha os procedimentos metodológicos que foram utilizados, a seção 4 apresenta os resultados e discussões e, por fim, a seção cinco apresenta as conclusões do estudo. As referências são listadas no final do artigo.

\section{FUNDAMENTAÇÃO TEÓRICA}

O lean manufacturing possui diversos princípios e ferramentas que contribuem para o sucesso das empresas que o implementam. Destaca-se primeiramente os cinco princípios básicos dessa filosofia: o valor; a cadeia de valor; o fluxo; a produção puxada; e a perfeição (WOMACK; JONES, 1998). A partir desses princípios, o lean manufacturing aumenta a eficiência da produção com a minimização de sete tipos básicos de desperdícios: Superprodução; Movimentação (de operadores, material ou máquina); Espera (de operador, material ou máquina); Transporte; Processamento inapropriado; Estoque e Defeitos (CHAUHAN; SINGH, 2012; MESSAGE COSTA et al., 2018; OHNO, 1997).

Entretanto, é importante que a implantação desse sistema seja realizada de forma personalizada. As organizações implementam esses princípios e ferramentas 
conforme suas necessidades e especificidades, a fim de maximizar os resultados obtidos. Dessa forma, existem diversas métricas que são utilizadas para avaliar o grau lean das empresas e, com isso, classificá-las em estágios de adoção do lean. A realização de estratégias de manufatura lean inapropriadas pode levar a um aumento de desperdícios, custos e tempo de produção. Assim, é necessário desenvolver uma metodologia adequada tanto para implementar estratégias lean apropriadas, como para avaliar a melhoria de desempenho contínua (ANVARI; ZULKIFLI; YUSUFF, 2013; KARIM; ARIF-UZ-ZAMAN, 2013).

As métricas lean são definidas como o processo contínuo que é responsável pelo aumento da produtividade na busca pela identificação e eliminação de desperdícios (KARIM; ARIF-UZ-ZAMAN, 2013; MARODIN et al., 2018). Tais métricas de avaliação devem possuir algumas propriedades básicas (SRINIVASARAGHAVAN; ALLADA, 2006): a) elas devem ser mensuráveis e estarem alinhadas com os objetivos estratégicos da empresa; b) elas devem permitir o controle e avaliação do desempenho; c) elas devem ajudar a compreender o cenário atual e ajudar na identificação de oportunidades de melhoria; d) elas devem ser atualizadas e realistas.

São muitas as métricas para a mensuração de sistemas lean. Para entendê-las melhor, foi feito um levantamento na literatura. De acordo com Ghosh (2012), algumas das principais métricas utilizadas na avaliação de projetos de lean manufacturing consistem na redução dos índices de estoque, sendo a gestão de estoques um meio utilizado para descrever uma eficiência de negócios quando tratando do estoque físico de produtos em relação à oferta e procura; eficiência na utilização do espaço físico, que mede a quantidade de espaço requerida na fabricação de um produto; produtividade dos funcionários, que mede a produção por hora de cada funcionário, uma vez que os funcionários devem ser qualificados para as suas tarefas e trabalhar de forma efetiva nas atividades que agregam valor aos clientes; custo de conversão associado a cada processo produtivo, que corresponde aos custos necessários para converter matérias-primas em produtos acabados; redução do tempo de espera industrial (fabricação em tempo de espera), que refere-se ao tempo entre o momento em que o cliente solicita um produto e o momento em que ele o recebe.

De acordo com Behrouzi e Wong (2013), algumas das métricas mais importantes estão relacionadas a taxas de rejeição. Dentre os itens desse grupo, 
destacam-se o percentual de produtos com defeito; o número de reclamações dos consumidores, uma vez que as queixas dos consumidores não são bons fatores para o processo produtivo, podendo ser originadas de: produtos com defeitos, atraso no período de entrega, mal atendimento; custo associado aos estoques existentes, que indica uma porcentagem do custo anual de estoques pelo número total de vendas anual; custo associado a transporte no ambiente produtivo, que indica uma porcentagem dos custos anuais de transporte pelo número total de vendas anual; tempo global das atividades não agregadoras de valores, que representa o tempo global das atividades não agregadoras de valores, sendo uma porcentagem do valor de tempo não agregado em relação ao tempo total.

Srinivasaraghavan e Allada (2006), por sua vez, apontam como importantes as métricas de redução na porcentagem de sucata em relação às vendas, que diz respeito a porcentagem de sucata gerada como uma porcentagem das vendas totais e o número de eventos kaizen realizados na empresa ao longo do ano. Tratam-se de eventos utilizados para identificar e resolver problemas específicos em diferentes áreas da organização.

Para Lacerda, Xambre e Alvelos (2016), a redução nos lead time e as melhorias no tempo de valor agregado (value added time) são outras métricas fundamentais para avaliação de sistemas lean. A primeira refere-se a um intervalo de tempo compreendido entre o início e o término de uma atividade, enquanto que a segunda refere-se ao tempo das operações que, de acordo com o cliente, agrega valor ao produto e para o quanto ele está disposto a pagar.

Em sua pesquisa, Agarwal, Shankar e Tiwari (2006) apontam as métricas de velocidade de entrega dos pedidos colocados como fundamentais na avaliação da maturidade de projetos de lean manufacturing. A importância dessa métrica, segundo os autores, é a viabilização da entrega de produtos just in time. Para identificar corretamente o nível das empresas com relação a esse item, os seguintes pontos devem ser considerados: índices de confiabilidade de entrega dos pedidos, medida pelo perfeito atendimento de pedidos e demonstra o grau em que um fornecedor é capaz de atender seus clientes dentro do prazo de entrega; e o comportamento do sistema produtivo frente à introdução de novos produtos, que corresponde a uma métrica que aproveita as melhores práticas aprendidas e padrões de qualidade 
reconhecidos, além de utilizar recursos altamente qualificados e equipamentos apropriados e da melhor forma.

Ainda com relação ao just in time, Behrouzi e Wong (2013) e Srinivasaraghavan e Allada (2006) apontam a necessidade de se avaliar o percentual de entregas "on time" (\%) e de entregas tardias, que medem a porcentagem de entregas no cronograma em um ano pelos fornecedores (o aumento das entregas "on time" e redução das entregas tardias está associado à melhoria do tempo de produção e à redução no número de reclamações dos consumidores) e o tempo de preparação de células ou linhas (setup), que indica uma porcentagem do tempo de setup em relação ao tempo total de produção.

Segundo Lacerda, Xambre e Alvelos (2016) e Srinivasaraghavan e Allada (2006) a métrica de robustez do sistema em função da variação do takt time é também precisa ser considerada. Ela corresponde a frequência em que um produto deve ser produzido para atender à demanda dos clientes e é dada pela seguinte fórmula: tempo de trabalho disponível por turno por taxa de demanda do cliente por turno. Aliado ao takt time, o chamado "first-pass correct output" pode contribuir para a verificação de maturidade de um sistema lean assim como a análise de oportunidades de melhoria. Isso deve-se ao fato de que o "first-pass correct output" representa o número de unidades que saem de um processo dividido pelo número de unidades que entram nesse processo ao longo de um período de tempo especificado (AGARWAL; SHANKAR; TIWARI, 2006; GHOSH, 2012).

De acordo com Wan e Chen (2008), o giro de estoque (inventory turn) e o manufacturing cycle time também precisam ser analisados. O primeiro é a taxa na qual uma operação ou negócio entrega ou vende produtos finalizados. É um indicador da eficiência do processo de planejamento de produção e de vendas / gerenciamento de marketing e é dado pela relação entre o custo dos bens vendidos pelo estoque médio. Já o segundo, serve de índice para redução de tempo de ciclo. Ele compara o tempo de valor adicionado com o tempo total de ciclo para mostrar a eficiência de um processo industrial.

Para Keyser e Sawhney (2013) e Arunagiri e Gnanavelbabu (2014) é essencial se avaliar a eficácia geral dos equipamentos (OEE) em projetos de lean manufacturing. É a melhor métrica prática que identifica o percentual de tempo de produção planejado 
que é de fato produtivo. Ela consiste em 3 métricas: disponibilidade, desempenho e qualidade. Uma pontuação OEE de 100\% representa uma produção perfeita. Os primeiros autores ainda consideram a métrica eficácia global do trabalho (OLE) imprescindível para se medir a eficácia do trabalho na força de trabalho. Ela se tornou uma ferramenta de gestão que é projetada para entender os efeitos que o desempenho da força de trabalho tem sobre o desempenho geral de fabricação.

Por fim, cita-se também a medida de desempenho continuo, utilizada para calcular a eficiência lean considerando tempo, custo, qualidade, flexibilidade e produtividade. Trata-se de uma métrica importante para avaliar sistemas lean (KARIM; ARIF-UZ-ZAMAN, 2013). O Quadro 1 apresenta a lista completa das métricas e referências que foram citadas anteriormente. Tais métricas foram utilizadas para a realização desta pesquisa.

Quadro 1 - Métricas utilizadas na avaliação de projetos de lean manufacturing encontradas na literatura

\begin{tabular}{|c|c|c|}
\hline $\mathbf{N}$ & Métricas a serem avaliadas & Fonte \\
\hline 1 & Redução dos índices de estoque & (GHOSH, 2012) \\
\hline 2 & Eficiência na utilização do espaço físico & $(\mathrm{GHOSH}, 2012)$ \\
\hline 3 & Produtividade dos funcionários & (GHOSH, 2012) \\
\hline 4 & Custo de conversão associado a cada processo produtivo & (GHOSH, 2012) \\
\hline 5 & $\begin{array}{l}\text { Redução do tempo de espera industrial (fabricação em } \\
\text { tempo de espera) }\end{array}$ & (GHOSH, 2012) \\
\hline 6 & Tempo para o "first-pass correct output" & (AGARWAL; SHANKAR; TIWARI, 2006; GHOSH, 2012) \\
\hline 7 & Redução nos lead time & (LACERDA; XAMBRE; ALVELOS, 2016) \\
\hline 8 & Melhorias no tempo de valor agregado (value added time) & (LACERDA; XAMBRE; ALVELOS, 2016) \\
\hline 9 & Robustez do sistema em função da variação do takt time & $\begin{array}{lcc}\text { (LACERDA; } & \text { XAMBRE; } & \text { ALVELOS, } \\
\text { SRINIVASARAGHAVAN; ALLADA, 2006) }\end{array}$ \\
\hline 10 & Redução no número de scrap & (BEHROUZI; WONG, 2013) \\
\hline 11 & Redução no número de reclamações dos consumidores & (BEHROUZI; WONG, 2013) \\
\hline 12 & Custo associado aos estoques existentes & (BEHROUZI; WONG, 2013) \\
\hline 13 & Custo associado a transporte no ambiente produtivo & (BEHROUZI; WONG, 2013) \\
\hline 14 & Tempo global das atividades não agregadoras de valores & (BEHROUZI; WONG, 2013) \\
\hline 5 & Tempo de preparação de células ou linhas (setup) & $\begin{array}{l}\text { (BEHROUZI; WONG, 2013; SRINIVASARAGHAVAN; } \\
\text { ALLADA, 2006) }\end{array}$ \\
\hline 16 & Redução na porcentagem de sucata em relação às vendas & (SRINIVASARAGHAVAN; ALLADA, 2006) \\
\hline 7 & $\begin{array}{l}\text { Aumento da porcentagem de entregas "on time" (\%) e } \\
\text { redução de entregas tardias }\end{array}$ & $\begin{array}{l}\text { (BEHROUZI; WONG, 2013; SRINIVASARAGHAVAN; } \\
\text { ALLADA, 2006) }\end{array}$ \\
\hline 18 & Aumento no número de eventos kaizen realizados & (SRINIVASARAGHAVAN; ALLADA, 2006) \\
\hline 19 & Velocidade de entrega dos pedidos colocados & (AGARWAL; SHANKAR; TIWARI, 2006) \\
\hline 20 & Índices de confiabilidade de entrega dos pedidos & (AGARWAL; SHANKAR; TIWARI, 2006) \\
\hline 21 & $\begin{array}{l}\text { Comportamento do sistema produtivo frente à introdução } \\
\text { de novos produtos }\end{array}$ & (AGARWAL; SHANKAR; TIWARI, 2006) \\
\hline 22 & OEE (eficácia geral dos equipamentos) & $\begin{array}{l}\text { (ARUNAGIRI; GNANAVELBABU, } 2014 ; \quad \text { KEYSER; } \\
\text { SAWHNEY, 2013) }\end{array}$ \\
\hline 23 & Eficácia global do trabalho (OLE) & (KEYSER; SAWHNEY, 2013) \\
\hline 24 & Medida de desempenho continuo & (KARIM; ARIF-UZ-ZAMAN, 2013) \\
\hline 25 & Giro de estoque (inventory turn) & (WAN; FRANK CHEN, 2008) \\
\hline 26 & Manufacturing cycle time & (WAN; FRANK CHEN, 2008) \\
\hline
\end{tabular}




\section{PROCEDIMENTOS METODOLÓGICOS}

Inicialmente, torna-se importante classificar a pesquisa apresentada por este artigo. Trata-se de uma pesquisa de natureza aplicada, pois busca gerar conhecimento para aplicação prática em projetos de implantação de sistemas lean. Para tanto será utilizada uma abordagem qualitativa e quantitativa, uma vez que este artigo toma por base notas de percepções de profissionais que lidam com sistemas produtivos lean e, posteriormente, é realizada uma análise estatística dos dados. Com relação aos objetivos, a pesquisa pode ser classificada como exploratória uma vez que a temática "maturidade de sistemas produtivos lean" ainda apresenta gaps a serem pesquisados e melhores explorados. Por fim, as estratégias de pesquisas utilizadas neste artigo foram pesquisa bibliográfica e survey (GIL, 2010; SILVA; MENEZES, 2005).

Para a análise das principais métricas utilizadas na avaliação da maturidade de projetos lean, foco deste estudo, foi realizada uma revisão sistemática da literatura. Para tanto, foram selecionados 81 artigos das seguintes bases científicas: Emerald Insight, ScienceDirect, Taylor \& Francis, Scientific e Springer Link. Para a realização das buscas, os foram utilizadas diferentes combinações dos seguintes termos: "lean", "manufacturing", "success", "evaluation", "tools", "difficulties", "implementation", entre outros. A partir desses artigos, foi possível a seleção das principais características, ferramentas, conceitos e princípios utilizados nos sistemas lean. A análise dos artigos selecionados também possibilitou a identificação das principais métricas utilizadas na avaliação da maturidade de sistemas lean, apresentadas anteriormente no Quadro 1.

\subsection{Elaboração do Questionário e Seleção da Amostra para a Coleta de Dados}

A elaboração do questionário foi feita tomando-se por base as métricas apresentadas no Quadro 1. O mesmo foi composto de 26 perguntas referentes a cada métrica a serem avaliadas em escalas de 1 a 10, por meio das quais o respondente deve assinalar o grau de aplicação da referida métrica no ambiente lean em que trabalha. Para sua montagem, foi escolhida a ferramenta Google Forms, um mecanismo prático e rápido para surveys. É importante salientar que tal questionário foi aprovado pelo Comitê de Ética em Pesquisas da Universidade. 
A seleção dos possíveis respondentes considerou pesquisadores em lean e profissionais de mercado e, dessa forma, foi possível listar 120 profissionais. O contato foi feito via e-mail, após um prazo de dois meses, foram recebidos 43 questionários. Pesquisadores em lean responderam ao questionário tomando por base aquilo que identificam nas empresas com as quais possuem contato. Profissionais de mercado responderam o questionário tomando por base a realidade de suas empresas. Em relação à amostra, dos 43 respondentes, 44\% trabalhavam com pesquisa; $14 \%$ eram do setor automotivo e $14 \%$ atuavam em consultoria. 0 restante da amostra pertencia a outros setores, como químico, metalúrgico, energético, construção civil, logística e comércio.

\subsection{Ferramentas Estatísticas Utilizadas}

Os dados coletados foram tabulados em uma planilha Excel. As análises estatísticas utilizadas foram: 1) análise de respondentes outliers por meio de Escalonamento Multidimensional (EMD) e 2) Ordenação das métricas por meio de um mecanismo utilizado pelo software SPSS para combinação linear de variáveis (empregado na Análise Fatorial Exploratória). Apresenta-se, nos itens a seguir, a descrição de cada técnica estatística utilizada.

O escalonamento multidimensional trata-se de uma técnica para a análise de dados de similaridade (ou dissimilaridade) entre um conjunto de objetos (BORG; GROENEN, 1997). Uma grande qualidade do EMD, frequentemente ressaltada pelos usuários da técnica, é sua robustez, ou seja, os métodos são capazes de recuperar a estrutura espacial dos dados mesmo em face de grandes perturbações nos dados originais (HAIR et al., 2009; LIN; FONG, 2019; SIBSON, 1979).

Para Fávero et al. (2009), o EMD apresenta alguns indicadores que indicam o nível e a qualidade do ajuste realizado, sendo os principais Stress (Standardized Residual Sum of Squares), SStress e RSQ ( $\left.{ }^{2}\right)$ (HAIR et al., 2009).

O Escalonamento Multidimensional foi utilizado nesta pesquisa para identificar os respondentes outliers e definir scores fatoriais para a ordenação das variáveis segundo seu grau de aplicação. Um respondente outlier pode ser entendido como aquele cuja resposta foge consideravelmente em relação à opinião média do grupo. 
Após a eliminação dos respondentes outliers, partiu-se para a ordenação das métricas e isso foi feito por meio da busca da melhor combinação linear para o conjunto de métricas. O objetivo não é realizar uma redução de escala como ocorre na AFE tradicional, mas apenas ordenar as variáveis (no presente caso as métricas). Em função disso, não se analisa tamanho de amostra, comunalidade, variância explicada pelo modelo, etc. Busca-se apenas um ajuste das variáveis pela melhor combinação linear, que neste caso é decorrente da extração do primeiro fator (FIELD, 2009). Tal análise foi feita por meio do software IBM SPSS Statistics 22. Este procedimento metodológico também pode ser observado na pesquisa de Cazeri et al. (2018), isto é, os autores supracitados também utilizaram a AFE para ordenar as variáveis.

\section{RESULTADOS}

A apresentação dos resultados foi realizada tomando-se por base a apresentação dos mapas espaciais obtidos pelo EMD e ordenação das variáveis segundo os scores fatoriais. Posteriormente, os resultados foram confrontados com a literatura.

\subsection{Resultados referentes ao Escalonamento Multidimensional}

A técnica EMD também foi aplicada aos dados coletados, obtendo-se os resultados apresentados na Figura 1 e Figura 2. Pode-se observar por meio da Figura 1 que todos os índices para Stress, Young's Stress e RSQ são adequados. A distribuição espacial dos respondentes gerada pelo software IBM SPSS 22 é apresentada pela Figura 2. 
Figura 1 - Resultados do EMD ajustado por distância euclidiana normal

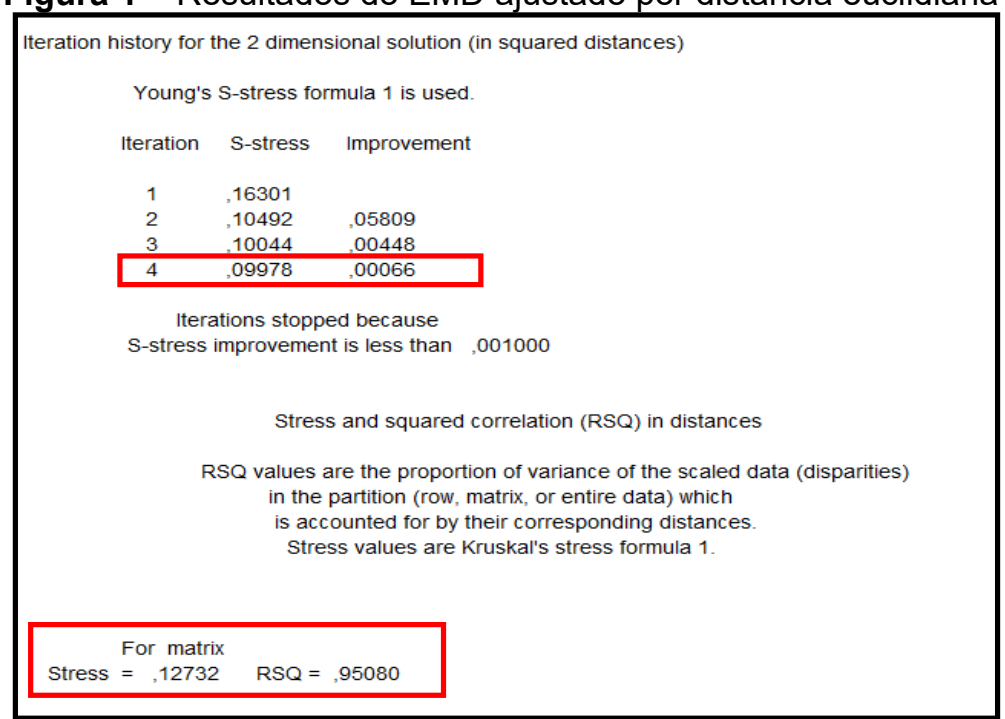

Fonte: Autores

Figura 2 - EMD referente aos 43 respondentes

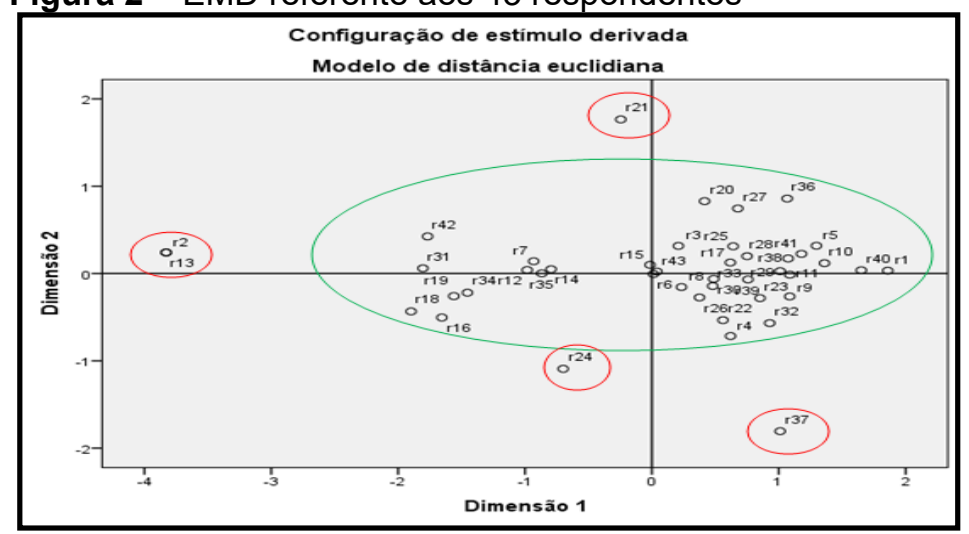

Fonte: Autores

É possível observar que dos 43 respondentes, cinco deles (r2, r13, r21, r24 e r37), demarcadas por círculos vermelhos, se encontram distantes em relação ao grupo principal, delineado por um círculo verde, e desta forma podem ser classificados como outliers. Isso significa que as respostas dadas por esses cinco respondentes não condizem com a opinião da maioria e, com isso, podem ser descartadas.

\subsection{Resultados referentes à ordenação das métricas}

Para a ordenação das variáveis utilizou-se novamente o software SPSS, sendo as métricas classificadas segundo os scores fatoriais. Quanto maior o valor de um score para uma determinada prática, mais esta prática se encontra relacionada a aquilo que está sendo estudado. Foi feita uma análise das 26 métricas apresentadas 
no Quadro 1 para uma amostra de 38 respondentes (a nova amostra é composta pela amostra original eliminando-se os outliers). O Quadro 2 apresenta o ranking das métricas tidas como mais aplicadas na avaliação da maturidade de projetos lean ordenadas segundo os scores obtidos.

Quadro 2 - Scores dos fatores para o critério aplicação

\begin{tabular}{|c|c|c|c|}
\hline \multicolumn{4}{|c|}{ GRAU DE APLICAÇÃO } \\
\hline Ranking & Score & Métrica & Descrição \\
\hline $1^{\circ}$ & 1,42599 & 3 & Produtividade dos funcionários \\
\hline $2^{\circ}$ & 1,26237 & 11 & Redução no número de reclamações dos consumidores \\
\hline $3^{\circ}$ & 1,2586 & 15 & Tempo de preparação de células ou linhas (setup) \\
\hline $4^{\circ}$ & 1,22659 & 7 & Redução nos lead time \\
\hline $5^{\circ}$ & 1,21809 & 1 & Redução dos índices de estoque \\
\hline $6^{\circ}$ & 0,95595 & 17 & $\begin{array}{l}\text { Aumento da porcentagem de entregas "on time" }(\%) \text { e } \\
\text { redução de entregas tardias }\end{array}$ \\
\hline $7^{\circ}$ & 0,87964 & 10 & Redução no número de scrap \\
\hline $8^{\circ}$ & 0,58672 & 5 & $\begin{array}{c}\text { Redução do tempo de espera industrial (fabricação em } \\
\text { tempo de espera) }\end{array}$ \\
\hline $9^{\circ}$ & 0,48373 & 25 & Giro de estoque (inventory turn) \\
\hline $10^{\circ}$ & 0,42923 & 12 & Custo associado aos estoques existentes \\
\hline $11^{\circ}$ & 0,30045 & 8 & Melhorias no tempo de valor agregado (value added time) \\
\hline $12^{\circ}$ & 0,28826 & 22 & OEE (eficácia geral dos equipamentos) \\
\hline $13^{\circ}$ & 0,27497 & 19 & Velocidade de entrega dos pedidos colocados \\
\hline $14^{\circ}$ & 0,18134 & 20 & Índices de confiabilidade de entrega dos pedidos \\
\hline $15^{\circ}$ & $-0,09077$ & 26 & Manufacturing cycle time \\
\hline $16^{\circ}$ & $-0,11371$ & 18 & Aumento no número de eventos kaizen realizados \\
\hline $17^{\circ}$ & $-0,2947$ & 2 & Eficiência na utilização do espaço físico \\
\hline $18^{\circ}$ & $-0,47583$ & 4 & Custo de conversão associado a cada processo produtivo \\
\hline $19^{\circ}$ & $-0,4925$ & 14 & Tempo global das atividades não agregadoras de valores \\
\hline $20^{\circ}$ & $-0,7936$ & 16 & Redução na porcentagem de sucata em relação às vendas \\
\hline $21^{\circ}$ & $-0,91941$ & 9 & Robustez do sistema em função da variação do takt time \\
\hline $22^{\circ}$ & $-1,15531$ & 13 & Custo associado a transporte no ambiente produtivo \\
\hline $23^{\circ}$ & $-1,1691$ & 6 & Tempo para o "first-pass correct output" \\
\hline $24^{\circ}$ & $-1,55357$ & 21 & $\begin{array}{l}\text { Comportamento do sistema produtivo frente à introdução de } \\
\text { novos produtos }\end{array}$ \\
\hline $25^{\circ}$ & $-1,7989$ & 23 & Eficácia global do trabalho (OLE) \\
\hline $26^{\circ}$ & $-1,91453$ & 24 & Medida de desempenho continuo \\
\hline
\end{tabular}

Fonte: Autores

É possível observar que as métricas de número 3, 11, 15, 7 e 1 formam um aglomerado e destacam-se pelos maiores scores, nessa ordem. Pode-se verificar que os scores caem gradativamente até sua inversão de sinal, na métrica 26. Posteriormente, os scores continuam diminuindo aos poucos até chegar em seu valor 
mínimo de -1,91453, identificado pela métrica 24. Pode-se notar também que das dez métricas mais aplicadas, quatro delas estão associadas ao critério tempo (lead time, tempo de espera industrial, tempo de preparação de células, tempo de entrega dos pedidos aos consumidores) e três estão associadas ao critério estoque (redução dos índices de estoque, redução dos custos associados aos estoques e giro de estoque). As demais correspondem à redução do número de reclamações dos consumidores, produtividade dos funcionários e redução do número de scraps.

\subsection{Debates dos Resultados}

A partir dos resultados acima apresentados, é possível realizar algumas observações. Primeiramente, é importante destacar que a métrica que obteve os maiores scores foi a produtividade dos funcionários, ou seja, trata-se de um indicador bastante aplicado, de acordo com a amostra pesquisada. Esse resultado vai de encontro à literatura, visto que tal indicador é apontado como relevante em pesquisas como a de Losonci e Demeter (2013) e Ghosh (2012). Na pesquisa de Ghosh (2012), a produtividade dos funcionários foi a que mais atingiu melhorias operacionais. Além disso, a importância dos funcionários para o bom funcionamento do lean é enfatizada por Pampanelli, Found e Bernardes (2014).

A redução no número de reclamações dos consumidores - segunda métrica mais utilizada -, por sua vez, vai diretamente ao encontro à preocupação central do lean: a geração de valor para os consumidores. Como o que não gera valor para o consumidor é considerado desperdício, a quantidade de reclamações dos consumidores é um importante indicador de falhas no sistema produtivo (BEHROUZI; WONG, 2013; WOMACK; JONES, 1998). Adicionalmente, de acordo com Ugochukwu, Engstrom e Langstrand (2013), dentre os principais benefícios que a filosofia lean gera está o aumento na satisfação dos clientes. Dessa forma, a correta implantação do lean, ao aumentar a satisfação dos consumidores, também reduz o número de reclamações dos mesmos.

Outras importantes fontes de desperdício para a filosofia lean que também se destacaram na pesquisa são o tempo de preparação de células ou linhas (setup) e os estoques. Tratam-se de indicadores que devem ser alvo de melhoria contínua a fim de aumentar a produtividade dos sistemas produtivos (BEHROUZI; WONG, 2013; 
BÜYÜKÖZKAN; KAYAKUTLU; KARAKADILAR, 2015; GHOSH, 2012). Ao contrário da produção em massa, que reduz custos produzindo em grandes quantidades, o lean visa fabricar produtos diversificados com um bom preço. Nesse sentido, a flexibilidade da empresa refere-se à capacidade de se adequar às variações na demanda dos clientes, sejam elas qualitativas ou quantitativas (CORIAT, 1994). Isso é possível devido à redução nos tempos de setup. A melhor maneira de se reduzir esses tempos de preparação de células/ linhas é por meio da troca rápida de ferramentas, também conhecida como SMED (Single Minute Exchange of Die), que permitem que o Just in Time seja um sistema eficaz (MESSAGE COSTA et al., 2018; WOMACK; JONES, 1998).

Por fim, o lead time também foi identificado na pesquisa como um indicador relevante. Por aferir o tempo total que a empresa leva desde o processamento de um pedido até a entrega deste pedido para o cliente, o lead time se caracteriza como um importante indicador da eficiência do sistema e da adesão desse sistema ao lean. Dentre as razões para isso, destaca-se o fluxo unitário de peças e o layout celular que, se bem implantados, possibilitam a redução do lead time (LACERDA; XAMBRE; ALVELOS, 2016; RAMPASSO; ANHOLON, 2017; ROTHER; SHOOK, 2012).

A partir das análises acima, é possível verificar que a literatura corrobora com os resultados obtidos no presente projeto de pesquisa, que apontam as métricas citadas acima como as mais aplicadas em um contexto empresarial de lean manufacturing.

\section{CONCLUSÕES}

O mercado atual é caracterizado pelo dinamismo e participação global gerando, desta forma, intensa competição entre as empresas na busca pela maior satisfação dos clientes e participação de mercado. Muitos são os benefícios observados em decorrência da adoção do lean production como, por exemplo, a redução dos custos de produção, o aumento da qualidade e a maior satisfação dos clientes finais (DEIF; ELMARAGHY, 2014; MARODIN et al., 2018; RAMPASSO; ANHOLON, 2017). Entretanto, como Dal Forno et al. (2014) e Jadhav, Mantha e Rane (2014) apontaram, apesar dos bons resultados observados na implementação da filosofia lean nas 
últimas décadas, ainda há muitas oportunidades de melhoria, o que justifica pesquisas adicionais nessa área.

Este artigo teve como objetivo identificar quais são as principais métricas utilizadas por empresas brasileiras na avaliação da maturidade de sistemas lean e mediante aos resultados apresentados, pode-se dizer que tal objetivo foi alcançado. Os resultados evidenciaram que as métricas julgadas como mais aplicadas nas empresas no Brasil estão relacionadas à produtividade dos funcionários, número de reclamações dos consumidores, tempo de setup, lead times e índice de estoques.

Dessa forma, o presente estudo mostra a importância das variáveis acima para o bom funcionamento do lean. É possível constatar que os resultados aqui apresentados confirmam o que é mostrado na literatura, isto é, de acordo com a amostra utilizada, no Brasil, as empresas focam em indicadores-chave do lean: produtividade dos funcionários, satisfação dos consumidores, lead time, setup e estoques.

Vale ressaltar o caráter exploratório desta pesquisa, visando ampliar o debate sobre o tema. Diferentes resultados podem ser obtidos a partir de diferentes amostras de respondentes. Os resultados decorrentes deste trabalho poderão ser utilizados por outros pesquisadores em suas futuras pesquisas e também por profissionais de mercado, para avaliação de suas empresas.

\section{AGRADECIMENTOS}

Ao Conselho Nacional de Desenvolvimento Científico e Tecnológico (CNPq), bolsas 307536/2018-1; 305442/2018-0; e 311530/2018-4.

A Coordenação de Aperfeiçoamento de Pessoal de Nível Superior (CAPES), processo 88887.464433/2019-00.

\section{REFERÊNCIAS}

AGARWAL, A.; SHANKAR, R.; TIWARI, M. K. Modeling the metrics of lean, agile and leagile supply chain: An ANP-based approach. European Journal of Operational Research, v. 173, n. 1, p. 211-225, 2006. https://doi.org/10.1016/j.ejor.2004.12.005

ANHOLON, R.; SANO, A. T. Analysis of critical processes in the implementation of lean manufacturing projects using project management guidelines. The International Journal of Advanced Manufacturing Technology, v. 84, n. 9-12, p. 2247-2256, 2016. https://doi.org/10.1007/s00170-015-7865-9 
ANVARI, A.; ZULKIFLI, N.; YUSUFF, R. M. A dynamic modeling to measure lean performance within lean attributes. The International Journal of Advanced Manufacturing Technology, v. 66, n. 5-8, p. 663-677, 2013. https://doi.org/10.1007/s00170-012-4356-0

ARUNAGIRI, P.; GNANAVELBABU, A. Identification of high impact lean production tools in automobile industries using weighted average method. Procedia Engineering, v. 97, p. 2072-2080, 2014. https://doi.org/10.1016/j.proeng.2014.12.450

BABALOLA, O.; IBEM, E. O.; EZEMA, I. C. Implementation of lean practices in the construction industry: A systematic review. Building and Environment, v. 148, n. October 2018, p. 34-43, 2018. https://doi.org/10.1016/j.buildenv.2018.10.051

BEHROUZI, F.; WONG, K. Y. An integrated stochastic-fuzzy modeling approach for supply chain leanness evaluation. International Journal of Advanced Manufacturing

Technology, v. 68, n. 5-8, p. 1677-1696, 2013. https://doi.org/10.1007/s00170-013-4966-1

BHASIN, S. Measuring the Leanness of an organisation. International Journal of Lean Six Sigma, v. 2, n. 1, p. 55-74, 2011. https://doi.org/10.1108/20401461111119459

BHASIN, S. Prominent obstacles to lean. International Journal of Productivity and Performance Management, v. 61, n. 4, p. 403-425, 2012.

https://doi.org/10.1108/17410401211212661

BORG, I.; GROENEN, P. Modern Multidimensional Scaling. New York, NY: Springer New York, 1997. https://doi.org/10.1007/978-1-4757-2711-1

BÜYÜKÖZKAN, G.; KAYAKUTLU, G.; KARAKADILAR, İ. S. Assessment of Lean Manufacturing Effect on Business Performance using Bayesian Belief Networks. Expert Systems with Applications, v. 42, n. 19, p. 6539-6551, 2015.

https://doi.org/10.1016/j.eswa.2015.04.016

CAZERI, G. T. et al. An assessment of the integration between corporate social responsibility practices and management systems in Brazil aiming at sustainability in enterprises. Journal of Cleaner Production, 2018. https://doi.org/10.1016/j.jclepro.2018.02.023

CHAUHAN, G.; SINGH, T. P. Measuring parameters of lean manufacturing realization. Measuring Business Excellence, v. 16, n. 3, p. 57-71, 2012. https://doi.org/10.1108/13683041211257411

CORIAT, B. Pensar pelo avesso: o modelo japonês de trabalho e organização. Rio de Janeiro: UFRJ/ Revan, 1994.

DEIF, A. M.; ELMARAGHY, H. Cost performance dynamics in lean production leveling. Journal of Manufacturing Systems, v. 33, n. 4, p. 613-623, out. 2014. https://doi.org/10.1016/j.jmsy.2014.05.010

FÁVERO, L. P. et al. Análise de dados: modelagem multivariada para tomada de decisões. [s.l.] Campus, 2009.

FIELD, A. Discovering Statistics Using SPSS. 3. ed. London: Sage Publications, 2009.

FORNO, A. J. D. et al. Value stream mapping: A study about the problems and challenges found in the literature from the past 15 years about application of Lean tools. International 
Journal of Advanced Manufacturing Technology, v. 72, n. 5-8, p. 779-790, 2014. https://doi.org/10.1007/s00170-014-5712-z

GELEI, A.; LOSONCI, D.; MATYUSZ, Z. Lean production and leadership attributes - the case of Hungarian production managers. Journal of Manufacturing Technology Management, v. 26 , n. 4, p. 477-500, 2015. https://doi.org/10.1108/JMTM-05-2013-0059

$\mathrm{GHOSH}, \mathrm{M}$. Lean manufacturing performance in Indian manufacturing plants. Journal of Manufacturing Technology Management, v. 24, n. 1, p. 113-122, 2012. https://doi.org/10.1108/17410381311287517

GIL, A. Como Elaborar Projetos de Pesquisa. São Paulo: Editora Atlas, 2010. HAIR, J. F. et al. Multivariate Data Analysis. 7. ed. [s.I.] Pearson, 2009.

JABBOUR, A. B. L. D. S.; JUNIOR, J. C. O.; JABBOUR, C. J. C. Extending lean manufacturing in supply chains: A successful case in brazil. Benchmarking: An International Journal, v. 21, n. 6, p. 1070-1083, 2014. https://doi.org/10.1108/BIJ-01-2013$\underline{0014}$

JADHAV, J. R.; MANTHA, S. S.; RANE, S. B. Exploring barriers in lean implementation. International Journal of Lean Six Sigma, v. 5, n. 2, p. 122-148, 2014. https://doi.org/10.1108/IJLSS-12-2012-0014

JING, S.; NIU, Z.; CHANG, P.-C. The application of VIKOR for the tool selection in lean management. Journal of Intelligent Manufacturing, p. 1-15, 14 set. 2015.

KARIM, A.; ARIF-UZ-ZAMAN, K. A methodology for effective implementation of lean strategies and its performance evaluation in manufacturing organizations. Business Process Management Journal, v. 19, n. 1, p. 169-196, 2013.

https://doi.org/10.1108/14637151311294912

KEYSER, R. S.; SAWHNEY, R. S. Reliability in lean systems. International Journal of Quality \& Reliability Management, v. 30, n. 3, p. 223-238, 2013.

https://doi.org/10.1108/02656711311299818

KHANCHANAPONG, T. et al. The unique and complementary effects of manufacturing technologies and lean practices on manufacturing operational performance. International Journal of Production Economics, v. 153, p. 191-203, 2014.

https://doi.org/10.1016/j.ijpe.2014.02.021

LACERDA, A. P.; XAMBRE, A. R.; ALVELOS, H. M. Applying Value Stream Mapping to eliminate waste: A case study of an original equipment manufacturer for the automotive industry. International Journal of Production Research, v. 54, n. 6, p. 1708-1720, 2016. https://doi.org/10.1080/00207543.2015.1055349

LEYER, M.; MOORMANN, J. How lean are financial service companies really? Empirical evidence from a large scale study in Germany. International Journal of Operations and Production Management, v. 34, n. 11, p. 1366-1388, 2014. https://doi.org/10.1108/IJOPM06-2013-0296

LIN, L.; FONG, D. K. H. Bayesian multidimensional scaling procedure with variable selection. Computational Statistics and Data Analysis, v. 129, p. 1-13, 2019.

https://doi.org/10.1016/j.csda.2018.07.007 
LOSONCI, D.; DEMETER, K. Lean production and business performance: international empirical results. Competitiveness Review: An International Business Journal incorporating Journal of Global Competitiveness, v. 23, n. 3, p. 218-233, 2013. https://doi.org/10.1108/10595421311319816

LUCATO, W. C. et al. Performance evaluation of lean manufacturing implementation in Brazil. International Journal of Productivity and Performance Management, v. 63, n. 5, p. 529-549, 2014. https://doi.org/10.1108/IJPPM-04-2013-0085

MARODIN, G. et al. Lean product development and lean manufacturing: Testing moderation effects. International Journal of Production Economics, v. 203, n. March, p. 301-310, 2018. https://doi.org/10.1016/j.ijpe.2018.07.009

MESSAGE COSTA, L. B. et al. Lean, six sigma and lean six sigma in the food industry: A systematic literature review. Trends in Food Science \& Technology, v. 82, n. August, p. 122-133, 2018. https://doi.org/10.1016/j.tifs.2018.10.002

OHNO, T. O Sistema Toyota de Produção: além da produção em larga escala. Porto Alegre: Bookman, 1997.

PAMPANELLI, A. B.; FOUND, P.; BERNARDES, A. M. A Lean \& Green Model for a production cell. Journal of Cleaner Production, v. 85, p. 19-30, 2014.

https://doi.org/10.1016/j.jclepro.2013.06.014

RAMPASSO, I. S.; ANHOLON, R. Parâmetros para avaliação de células de manufatura que utilizam a filosofia lean: uma revisão da literatura. Revista Produção Online, v. 17, n. 4, p. 1329, 15 dez. 2017. https://doi.org/10.14488/1676-1901.v17i4.2637

ROTHER, M.; SHOOK, J. Aprendendo a enxergar: mapeando o fluxo de valor para agregar valor e eliminar o desperdício. São Paulo: Lean Institute Brasil, 2012.

SIBSON, R. Studies in the Robustness of Multidimensional Scaling: Perturbational Analysis of Classical Scaling. Journal of the Royal Statistical Society. Series B (Methodological), v. 41, n. 2, p. 217-229, 1979. https://doi.org/10.1111/j.2517-6161.1979.tb01076.x

SILVA, E. L.; MENEZES, E. M. Metodologia da Pesquisa e Elaboração de Dissertação - 4a edição. Portal, p. 138p, 2005.

SILVA, S. K. P. N.; PERERA, H. S. C.; SAMARASINGHE, G. D. Viability of Lean Manufacturing Tools and Techniques in the Apparel Industry in Sri Lanka. Applied Mechanics and Materials, v. 110-116, p. 4013-4022, 2012.

https://doi.org/10.4028/www.scientific.net/AMM.110-116.4013

SRINIVASARAGHAVAN, J.; ALLADA, V. Application of mahalanobis distance as a lean assessment metric. International Journal of Advanced Manufacturing Technology, v. 29, n. 11-12, p. 1159-1168, 2006. https://doi.org/10.1007/s00170-005-0004-2

UFUA, D. E.; PAPADOPOULOS, T.; MIDGLEY, G. Systemic Lean Intervention: Enhancing Lean with Community Operational Research. European Journal of Operational Research, v. 268, n. 3, p. 1134-1148, 2018. https://doi.org/10.1016/j.ejor.2017.08.004

UGOCHUKWU, P.; ENGSTROM, J.; LANGSTRAND, J. Lean In The Supply Chain: A 
Literature Review. Management and Production Engineering Review, v. 3, n. 4, p. 87-96, 2013.

WAN, H. DA; FRANK CHEN, F. A leanness measure of manufacturing systems for quantifying impacts of lean initiatives. International Journal of Production Research, v. 46, n. 23, p. 6567-6584, 2008. https://doi.org/10.1080/00207540802230058

WOMACK, J. P.; JONES, D. T. A mentalidade enxuta nas empresas: elimine o desperdício e crie riqueza. Rio de Janeiro: Campus, 1998.

ZHOU, B. Lean principles, practices, and impacts: a study on small and medium-sized enterprises (SMEs). Annals of Operations Research, v. 241, n. 1-2, p. 457-474, 6 jun. 2016. https://doi.org/10.1007/s10479-012-1177-3

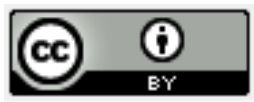

Artigo recebido em: 22/11/2018 e aceito para publicação em: 04/03/2020

DOI: http://dx.doi.org/10.14488/1676-1901.v20i1.3470 